\title{
Planung, Vorbereitung und Kontrolle bei traumatisch bedingten Eingriffen im Bereich der BWS und LWS
}

\author{
Jens Adermann, Christian Schmidt, Christoph Josten
}

\section{Zusammenfassung}

Die operative Behandlung von Frakturen der BWS und LWS wurde aufgrund einer zunehmenden Inzidenz der Frakturen in den vergangenen Jahren zunehmend wichtiger. Die Behandlung ist komplex und an hohe personelle und technische Anforderungen gebunden. Die existierenden Therapieempfehlungen müssen individuell auf den Patienten, sein biologisches Alter, seine Knochenqualität, sein Aktivitätslevel und seine persönlichen Ansprüche abgestimmt werden. Bei der operativen Versorgung stehen dorsale und ventrale Verfahren sowie eine kombinierte dorsoventrale Versorgung zur Verfügung. Ein modernes Versorgungskonzept sollte eine intraoperative Navigation, thorakoskopisch unterstützte Zugänge zur ventralen Säule, minimalinvasive perkutane Operationszugänge und die Möglichkeit der simultanen dorsoventralen Versorgung umfassen. Der Artikel soll einen Überblick über die Planung und Vorbereitung von Eingriffen im Bereich der verletzten BWS und LWS geben und intraoperative Kontrollinstrumente wie die Navigation und das Neuromonitoring vorstellen.
Planning, Preparation and Control of Surgical Interventions due to Trauma in the Region of the Thoracic and Lumbar Spines

Because of the rising incidence of fractures in the past few years, the surgical treatment of fractures of the thoracic and lumbar spine is gaining increasing importance. The treatment is complicated and associated with high personal and technical requirements. The currently valid therapeutic recommendations must be individually adapted to the patient, his/her biological age, bone quality, level of physical activity, and personal expectations. Both dorsal and ventral as well as combined dorsoventral approaches are available for surgical treatment. A modern management concept should encompass intraoperative navigation, thoroscopicassisted approaches to the ventral spine, minimally invasive percutaneous surgical approaches and the possibility for a simultaneous dorsoventral procedure. The present article presents a survey on the planning and preparation for operations in the region of injured thoracic and lumbar spines and intraoperative control techniques such as navigation and neuromonitoring.

\section{Einleitung}

Frakturen der Brust- und Lendenwirbelsäule spielen im klinischen Alltag aufgrund ihrer Häufigkeit und aufgrund der Morbidität und Mortalität eine wichtige Rolle. Die Inzidenz von Wirbelkörperfrakturen wird in Deutschland mit 50/100000, das Auftreten von neurologischen Ausfällen mit 3/100000 angege-

OP-JOURNAL 2011; 27: 42-52

(c) Georg Thieme Verlag KG Stuttgart · New York DOI http://dx.doi.org/10.1055/s-0030-1271015

ben. Dabei entfallen 15-37\% auf den Lumbalbereich und 15-24\% auf den Thorakalbereich [45]. Der thorakolumbale Übergang ist bei $67 \%$ der BWS- und LWS-Frakturen betroffen [34]. Ursächlich hierfür ist der Übergang von der Kyphose zur Lordose und von der durch den Brustkorb fixierten zur frei stehenden Wirbelsäule. 3-6\% der skeletalen Verletzungen sind Wirbelkörperfrakturen [26].

Die Zahlen zur Inzidenz schwanken je nach Studie zwischen 23 und 90/100000. Insgesamt ist mit ca. 8000 schwerwie- genden Verletzungen der BWS und LWS in Deutschland pro Jahr zu rechnen. Die Inzidenz ist altersabhängig und beträgt bei den unter 60-Jährigen nur 13/100000. Unter 60 Jahren sind Männer doppelt so häufig betroffen, über 60 Jahre ist die Mehrzahl der Patienten weiblich [19]. Dies ist bedingt durch die Dominanz der osteoporotischen Wirbelkörperfrakturen im höheren Lebensalter. Man geht in Deutschland von ca. 7,8 Mio. Osteoporoseerkrankten zwischen 50 und 79 Jahren aus, was einer Inzidenz von $26 \%$ in dieser Bevölkerungsgruppe entspricht. Osteoporotisch bedingte Wirbelkörperfrakturen stellen nicht selten die Erstmanifestation der Erkrankung dar. In Deutschland treten schätzungsweise 100000 osteoporotische Sinterungsfrakturen der Wirbelsäule pro Jahr auf [30].

Insgesamt findet sich in den vergangenen Jahrzehnten eine steigende Inzidenz von Wirbelkörperfrakturen zum einen aufgrund zunehmender Freizeitaktivitäten im Hochrasanzbereich und zum anderen aufgrund einer Zunahme der Mobilität im Alter [16]. Die Hauptverletzungsursachen sind Bagatelltraumen bei Osteoporosepatienten, Verkehrsunfälle, Stürze aus großer Höhe und Sportverletzungen. Häufig stehen Patienten unter Alkohol- oder Drogeneinfluss. Suizidale Verletzungen spielen ebenfalls eine große Rolle. Schuss- und Stichverletzungen finden sich in Deutschland im Vergleich zu anderen Ländern seltener als Ursache.

\section{Klassifikation}

Grundlage für eine erfolgreiche Therapie ist ein alltagstaugliches Klassifikationssystem. Denn nur durch eine exakte Einteilung lassen sich die verschiedenen Behandlungskonzepte miteinander vergleichen und Therapieleitlinien entwickeln.

Die Grundlagen der heute verwendeten Klassifikationen sind das 2-Säulen-Modell [48] und das 3-Säulen-Modell [49]. 
Basierend auf diesen Überlegungen entwickelten Magerl et al. 1994 die heute am weitesten verbreitete Klassifikation von Wirbelsäulenfrakturen der BWS und LWS [27]. Diese Einteilung wurde von der Arbeitsgemeinschaft für Osteosynthesefragen $(\mathrm{AO})$ übernommen und weiterentwickelt. Es werden A-, B- und C-Verletzungen unterschieden. Tab. 1 zeigt die Klassifikation.

\section{Therapieplanung}

Bei der Anamnese, Untersuchung und Diagnostik gilt, dass ein Patient mit Verdacht auf eine Wirbelkörperfraktur bis zum Beweis des Gegenteils als Wirbelsäulenverletzter anzusehen ist und entsprechende Stabilisierungsmaßnahmen vorzunehmen sind.

Ein Patient mit Verdacht auf eine Wirbelkörperfraktur ist bis zum Beweis des Gegenteils als Wirbelsäulenverletzter anzusehen.

\section{Anamnese}

Bei wachen und kooperativen Patienten erfolgt zu Beginn der Abklärung ein Anamnesegespräch. Dies hat einen hohen Stellenwert [46], da anhand der vom Patienten geäußerten Beschwerden und des Unfallmechanismus das weitere Vorgehen und die Wahl der Bildgebung festgelegt werden können.

Ein besonderes Augenmerk muss der behandelnde Arzt dabei auf den Unfallmechanismus legen. Bei Stürzen spielen die Fallhöhe und der Untergrund eine entscheidende Rolle, bei Sport- und Verkehrsunfällen ist die Rasanz von Bedeutung. Wenn möglich, sollte genau erfragt werden, ob es sich um einen Stauchungs-, Flexions- oder Hyperextensionsmechanismus handelte.

Um sich ein umfassendes Bild machen zu können, sollten neben dem Patienten auch der Notarzt, Rettungsdienstmitarbeiter und Angehörige des Patienten befragt werden.

Viele Patienten mit Wirbelkörperfrakturen sind jedoch aufgrund eines demenziellen Syndroms, wegen Alkohol- oder Drogeneinnahme oder infolge einer Analgosedierung nicht auskunftsfähig. Stehen keine verlässlichen Personen für eine Auskunft zur Verfügung, muss bei folgenden Zeichen bis zum Beweis des Gegenteils von einer Wirbelsäulenverletzung ausgegangen werden:

Tab. 1 Klassifikation.

\begin{tabular}{|c|c|c|}
\hline \multirow[t]{2}{*}{ A } & \multirow[t]{3}{*}{ A1 Impaktionsbruch } & A1.1 Deckplattenimpression \\
\hline & & A1.2 Keilbruch \\
\hline & & A1.3 Impaktionsbruch \\
\hline & \multirow[t]{3}{*}{ A2 Spaltbruch } & A2.1 sagittaler Spaltbruch \\
\hline & & A2.2 frontaler Spaltbruch \\
\hline & & A2.3 Kneifzangenbruch \\
\hline & \multirow[t]{3}{*}{ A3 Berstungsbruch } & A3.1 inkompletter Berstungsbruch \\
\hline & & A3.2 Berstungsspaltbruch \\
\hline & & A3.3 kompletter Berstungsbruch \\
\hline \multirow[t]{8}{*}{ B } & \multirow[t]{2}{*}{$\begin{array}{l}\text { B1 transligamentäre Flexions- } \\
\text { distraktionsverletzung }\end{array}$} & $\begin{array}{l}\text { B1.1 transligementäre Flexionsdistraktions- } \\
\text { verletzung mit Diskuszerreißung }\end{array}$ \\
\hline & & $\begin{array}{l}\text { B1.2 transligamentäre Flexionsdistraktions- } \\
\text { verletzung mit Korpusfraktur }\end{array}$ \\
\hline & \multirow{3}{*}{$\begin{array}{l}\text { B2 transossäre Flexionsdistraktions- } \\
\text { verletzung }\end{array}$} & B2.1 horizontale Wirbelzerreißung \\
\hline & & $\begin{array}{l}\text { B2.2 Flexionsspondylolyse mit } \\
\text { Bandscheibenzerreißung }\end{array}$ \\
\hline & & B2.3 Flexionsspondylolyse mit Korpusfraktur \\
\hline & \multirow[t]{3}{*}{ B3 Hyperextensionsscherverletzung } & B3.1 Hyperextensionssubluxation \\
\hline & & B3.2 Hyperextensionsspondylolyse \\
\hline & & B3.3 hintere Luxation \\
\hline \multirow[t]{7}{*}{ C } & \multirow[t]{3}{*}{ C1 Rotations-/Kompressionsverletzung } & C1.1 Rotationskeilbruch \\
\hline & & C1.2 Rotationsspaltbruch \\
\hline & & C1.3 Rotationsberstungsbruch \\
\hline & \multirow[t]{3}{*}{ C2 Rotations-/Distraktionsverletzung } & C2.1 Rotation mit B1-Verletzung \\
\hline & & C2.2 Rotation mit B2-Verletzung \\
\hline & & C2.3 Rotation mit B3-Verletzung \\
\hline & \multirow[t]{2}{*}{ C3 Rotations-/Scherverletzung } & C3.1 Slice-Fraktur \\
\hline & & C3.2 Rotationsschrägbruch \\
\hline
\end{tabular}

- Polytrauma (34\% der Polytraumapatienten haben Wirbelsäulenverletzungen [46])

- bewusstloser Patient

- unklare neurologische Symptome

- Wirbelsäulenschmerzen

- Unfallmechanismus (Hochrasanztrauma)

Bedingt durch den Schichtdienst in Krankenhäusern ist eine personelle Kontinuität des ärztlichen und pflegerischen Personals über einen längeren Zeitraum meistens nicht gegeben. Das Anamnesegespräch ist daher ebenso wie alle Untersuchungsergebnisse peinlich genau $\mathrm{zu}$ protokollieren. Nicht selten können kleine Veränderungen der Symptome im zeitlichen Verlauf zu einer Operationsindikation führen.
Eine exakte Protokollierung aller Untersuchungsergebnisse ist bei der Behandlung von Wirbelsäulenverletzten notwendig.

\section{Klinische Untersuchung}

Die klinische Untersuchung wird grundsätzlich am entkleideten Patienten durchgeführt. Um Manipulationen zu vermeiden, sind Patienten mit Verdacht auf eine instabile Wirbelsäulenverletzung durch geeignete Rettungsscheren von der Kleidung zu befreien. Dem Schamgefühl des Patienten sollte auch in der Primärdiagnostik Rechnung getragen werden und durch entsprechende räumliche Vorkehrungen eine Untersuchung außerhalb der Sichtweite von unbeteiligten Personen ermöglicht wer- 
den. Da verunfallte Patienten nicht selten unter Schock stehen, ist eine einfühlsame Untersuchung mit Erklärung der einzelnen Schritte unerlässlich.

Bei der Inspektion muss auf äußere Verletzungen, Hämatome und Schwellungen sowie auf Strukturauffälligkeiten und Fehlstellungen der Wirbelsäule geachtet werden. Die meisten Wirbelsäulenfrakturen gehen jedoch mit einem unauffälligen Inspektionsbefund einher. Grundsätzlich sollten auch schwierig zugängliche Körperpartien, insbesondere der Rücken, inspiziert werden. Bei Verdacht auf eine instabile Fraktur und entsprechender Lagerung des Patienten in einer Vakuummatratze werden solche Manipulationen jedoch zunächst unterlassen und die Bildgebung abgewartet, um iatrogene Frakturdislokationen und Rückenmarksverletzungen $\mathrm{zu}$ vermeiden.

Pei der manuellen Untersuchung liegt der Fokus auf Druck- und Klopfschmerzen im Bereich der Wirbelsäule. Außerdem können die Dornfortsätze getastet und evtl. Kontinuitätsunterbrechungen bemerkt werden.

Besteht kein Verdacht auf eine Instabilität, kann die Beweglichkeit der Wirbelsäule überprüft werden.

Auch bei klinisch führendem Verdacht auf eine Wirbelsäulenverletzung der BWS oder LWS muss präzise nach Begleitverletzungen, insbesondere nach Zeichen einer HWS-Verletzung oder eines Schädel-Hirn-Traumas gesucht werden

Den wichtigsten Stellenwert nimmt bei Wirbelsäulenverletzten die Erhebung des neurologischen Status ein. Bei Bund C-Verletzungen sowie multiplen Wirbelkörperfrakturen besteht ein höheres Risiko von neurologischen Ausfällen. Außerdem ist das Risiko bei BWSVerletzungen höher als bei Verletzungen des thorakolumbalen Übergangs oder der LWS [34].

Wenn möglich, wird ein erster Status bereits vom erstversorgenden Notarzt an der Unfallstelle vor Begin einer Analgosedierung erhoben.

Grundsätzlich muss jeder Arzt, der wirbelsäulenverletzte Patienten behandelt, imstande sein, einen genauen neurologischen Status zu erheben. Im Zweifel sollte jedoch zusätzlich ein Neurologe kon-

Tab. 2 Kennmuskeln zur Eruierung der Höhe der Schädigung mit entsprechenden Reflexen.

\begin{tabular}{lll}
$\begin{array}{l}\text { Läsions- } \\
\text { höhe }\end{array}$ & Parese & $\begin{array}{l}\text { Reflex- } \\
\text { verlust }\end{array}$ \\
\hline L3 & M. quadriceps femoris, M. iliopsoas (Hüftbeuger, Kniestrecker) & PSR \\
\hline L4 & M. quadriceps femoris, M. tibialis anterior (Hüftbeuger, Kniestrecker) & PSR \\
\hline L5 & M. extensor hallucis longus, M. extensor digitorum brevis (Fußheber) & TPR \\
\hline S1 & M. peronaeus, M. triceps surae (Fußsenker) &
\end{tabular}

sultiert werden. Auch bei neurologisch vorerkrankten Patienten mit Symptomen, welche bereits vor dem Unfall bestanden, ist zur genauen Abklärung einer neuen Komponente ein Neurologe hinzuzuziehen.

Bei Verletzungen der Brust- und Lendenwirbelsäule liegt der Fokus der Untersuchung auf der unteren Extremität. Die Kraftgrade aller Muskelgruppen werden geprüft und nach Janda angegeben. Die Kenntnis der Kennmuskeln (Tab. 2) ist wichtig, um bei einem Ausfall die Höhe der Schädigung abzuschätzen. Bei der Prüfung der Sensibilität hat sich in der Primärdiagnostik eine Prüfung der Oberflächensensibilität und Nozizeption bewährt. Anhand der Dermatome kann in der Regel die Höhe eines sensiblen Querschnitts sicher diagnostiziert werden. Des Weiteren ist eine Prüfung des Reflexstatus und des Sphinktertonus durchzuführen. Das Ergebnis der neurologischen Untersuchung wird auf entsprechenden Anamnesekarten dokumentiert [9] und das Gesamtergebnis mittels des FrankleScores zusammengefasst.

Im Verlauf der weiteren Diagnostik und Behandlung müssen engmaschige Kontrolluntersuchungen erfolgen, um evtl. Verschlechterungen frühzeitig zu diagnostizieren und entsprechende therapeutische Schritte einzuleiten.

Während des Klinikaufenthalts sollten engmaschige Kontrolluntersuchungen erfolgen, um evtl. Verschlechterungen frühzeitig zu diagnostizieren und entsprechende therapeutische Schritte einzuleiten.

Frankle-Score:

- A komplett = keine sensible oder motorische Funktion

- $\mathrm{B}$ inkomplett = Sensibilität erhalten, keine motorische Funktion unterhalb der Läsion bis in die sakralen Segmente

- $\mathrm{C}$ inkomplett = Sensibilität erhalten, motorische Funktion mit Kraftentfal- tung $<3 / 5$ unterhalb der Läsion bis in die sakralen Segmente

- D inkomplett = Sensibilität erhalten, motorische Funktion mit Kraftentfaltung $>3 / 5$ unterhalb der Läsion bis in die sakralen Segmente

- E normal = normale sensible und motorische Funktion

Messung der Muskelkraft nach Janda:

- $0=$ keine Muskelkontraktion sichtbar, keine Muskelaktivität

- 1 = sichtbare Muskelkontraktion, aber kein Bewegungseffekt

- 2 = schwache Muskelbewegung nur unter Aufhebung der Schwerkraft

- 3 = Anheben gegen Schwerkraft möglich

- 4 = Bewegung gegen leichten Widerstand möglich

$-5=$ normale Kraft

\section{Bildgebung}

Eine adäquate Bildgebung ist fester Bestandteil jeder Diagnostik von Wirbelsäulenverletzungen. Die klinische Untersuchung alleine ist nicht ausreichend für die Diagnosestellung [18]. Bei der Auswertung der angefertigten Bilder muss mit höchster Sorgfalt gearbeitet werden, weil übersehene Frakturen eine erhebliche Gefahr für den Patienten darstellen. Insgesamt fordert die Bildgebung der Wirbelsäule eine hohe interdisziplinäre Zusammenarbeit mit den Kollegen der Radiologie.

Die Diagnostik sollte idealerweise als Stufendiagnostik aufgebaut werden. Begonnen wird dabei mit einfach durchzuführenden, nicht invasiven und kostengünstigen Verfahren.

Klinisch bewährt hat sich ein Stufenschema aus konventioneller Röntgendiagnostik, Computertomografie und Magnetresonanztomografie. Bei polytraumatisierten Patienten hat sich aus zeitlichen Erwägungen heraus die Detektion mittels der CT-Polytraumaspirale als Standard etabliert. Nur in Sonderfäl- 
len werden konventionelle Röntgenaufnahmen der Wirbelsäule durchgeführt.

Die konventionellen Röntgenaufnahmen haben jedoch trotz der zunehmenden Verbreitung von CT und MRT nicht an Relevanz verloren. Sie sind sowohl im ambulanten als auch im stationären Bereich nahezu überall verfügbar, kostengünstig und mit einer niedrigen Strahlenbelastung für den Patienten einhergehend. Bei Verdacht auf Frakturen der Brustund Lendenwirbelsäule werden die BWS und LWS jeweils in 2 Ebenen aufgenommen. Wenn möglich, sollten die Aufnahmen im Stehen durchgeführt werden [43]. Instabilitätszeichen sind eine Kompression der vorderen Säule um mehr als $50 \%$, eine Kyphosierung um mehr als $11^{\circ}$ im Vergleich zu den Nachbarsegmenten, ein knöcherner Ausriss des vorderen Längsbands, Konturunregelmäßigkeiten der Wirbelkörperhinterkante, eine Höhenminderung an der Hinterkante, eine Translation der Hinterkante, eine Facettenverschiebung, eine Facettenluxation oder eine Facettenversetzung [21].

Die konventionellen Röntgenaufnahmen haben trotz der zunehmenden Verbreitung von CT und MRT nicht an Relevanz verloren.

Ergibt sich im konventionellen Röntgenbild der Verdacht auf eine Wirbelkörperfraktur, führen wir eine Computertomografie des entsprechenden Wirbelsäulenabschnitts durch. Wenn im konventionellen Röntgen keine Beteiligung der Hinterkante sichtbar ist, kein neurologisches Defizit besteht und eine spontane Mobilisierung möglich ist, kann unter Umständen auf die Anfertigung eines CTs verzichtet werden.

Das CT hat eine hohe Sensitivität und Spezifität bei der Diagnostik von Wirbelsäulenfrakturen und eine niedrigere Rate falscher Frakturklassifikationen als das konventionelle Röntgen [17].

Für die Operationsplanung sollte darauf geachtet werden, dass mindestens der nach kranial und kaudal angrenzende Wirbelkörper mit abgebildet wird. Idealerweise werden Rekonstruktionen in der Sagittalebene und Frontalebene durchgeführt. Es konnte aber auch gezeigt werden, dass ein Thorax-/Abdomen-/Becken-CT mit $5 \mathrm{~mm}$-Schnitten ohne Rekonstruktionen für eine sichere Diagnostik ausreicht [28].
Die Magnetresonanztomografie hat bei der Versorgung polytraumatisierter Patienten eine untergeordnete Rolle. Sie nimmt jedoch einen wesentlichen Stellenwert bei der Abklärung von unklaren neurologischen Symptomen ein. Außerdem bietet sie die Möglichkeit einer Abschätzung des Frakturalters. In der T2-STIR-Sequenz lässt sich 8-12 Wochen nach Fraktur ein Knochenödem nachweisen. Dies spielt v.a. bei der Erstellung eines Therapieregimes bei multiplen osteoporotischen Sinterungsfrakturen eine große Rolle.

Obwohl das MRT bei der Darstellung der Weichteile dem CT deutlich überlegen ist, ist die Diagnose von Verletzungen des posterioren Bandapparats mit einer Signifikanz von 79-90\% und einer Spezifität von 53-65\% noch nicht zufriedenstellend [42]. Häufig können mit epiduralen Hämatomen und Weichteilödemen nur indirekte Zeichen von Verletzungen des posterioren Bandapparats nachgewiesen werden [23].

Zusätzliche Untersuchungen wie Myelografie, Diskografie, Angiografie und Angio-CT können nach individueller Entscheidung durchgeführt werden, bleiben jedoch speziellen Fragestellungen vorbehalten [43]. Funktionsaufnahmen der BWS und LWS haben in der Akutphase keinen Stellenwert.

Bei der Wahl des geeigneten radiologischen Verfahrens muss der behandelnde Arzt immer auch an mögliche Begleitverletzungen denken. So ist bei BWS-Verletzungen ein CT des Thoraxes [15] und bei Verletzungen der LWS eine Abdomensonografie [9] immer indiziert.

\section{OP-Indikation, strategische Überlegungen, Zugangsplanung}

Aufgrund zahlreicher publizierter Studien ist es in den vergangenen Jahren gelungen, einheitliche Therapiekonzepte für die Behandlung von Wirbelkörperfrakturen aufzustellen, welche eine hohe Akzeptanz genießen. Die Therapieempfehlungen müssen jedoch stets auf den Patienten, sein biologisches Alter, seine Knochenqualität, sein Aktivitätslevel und nicht zuletzt auf seine persönlichen Ansprüche abgestimmt werden [43].

Die Therapieempfehlungen müssen auf den Patienten, sein biologisches Alter, seine Knochenqualität, sein Aktivitätslevel und seine persönlichen Ansprüche abgestimmt werden.
Konservative Therapie

Ein Teil der Wirbelkörperfrakturen, v.a. A1- und A2-Frakturen, können konservativ behandelt werden. Zu der frühfunktionellen Behandlung gehören dabei eine adäquate Schmerztherapie, eine frühzeitige rückengerechte Mobilisierung unter physiotherapeutischer Anleitung mit schmerzorientierter Vollbelastung sowie röntgenologische Therapiekontrollen.

\section{Operative Therapie}

Ziele der Operation sind die Reposition der Fehlstellung, die Retention der reponierten Stellung, die Dekompression des Spinalkanals, die Verhinderung neurologischer Defizite und die Rekonstruktion der vorderen Säule [9].

Prinzipiell ist die Wirbelsäule über dorsale und ventrale Zugänge operativ erreichbar. Zu den dorsalen Verfahren gehören die dorsale Instrumentierung, die knöcherne Dekompression des Spinalkanals sowie die Spondylodese durch eine Entknorpelung der kleinen Wirbelgelenke und die Anlagerung von allogenem oder autologem Knochenmaterial. Außerdem kann über den dorsalen $\mathrm{Zu}$ gang eine instrumentelle Aufrichtung der Kyphose erfolgen (Ligamentotaxis, Abb. 1).

$\mathrm{Zu}$ den ventralen Operationstechniken werden die ventrale Instrumentierung sowie die Korpektomie und die Implantation von Cages oder autologem oder allogenem Knochentransplantat gezählt. Mit dem TLIF und der Kyphoplastie stehen auch Operationstechniken zur Verfügung, die eine Stabilisierung der ventralen Säule über dorsale Zugänge ermöglicht.

Aufgrund der Knochenqualität und der Frakturmorphologie bestehen grundsätzliche Unterschiede in der Behandlung von Frakturen bei ansonsten gesunden Patienten gegenüber Frakturen bei Osteoporosepatienten. Daher werden im Folgenden die Therapiestrategien für beide Frakturtypen getrennt voneinander skizziert.

Ziele der Operation sind die Reposition der Fehlstellung, die Retention der reponierten Stellung, die Dekompression des Spinalkanals, die Verhinderung neurologischer Defizite und die Rekonstruktion der vorderen Säule. 


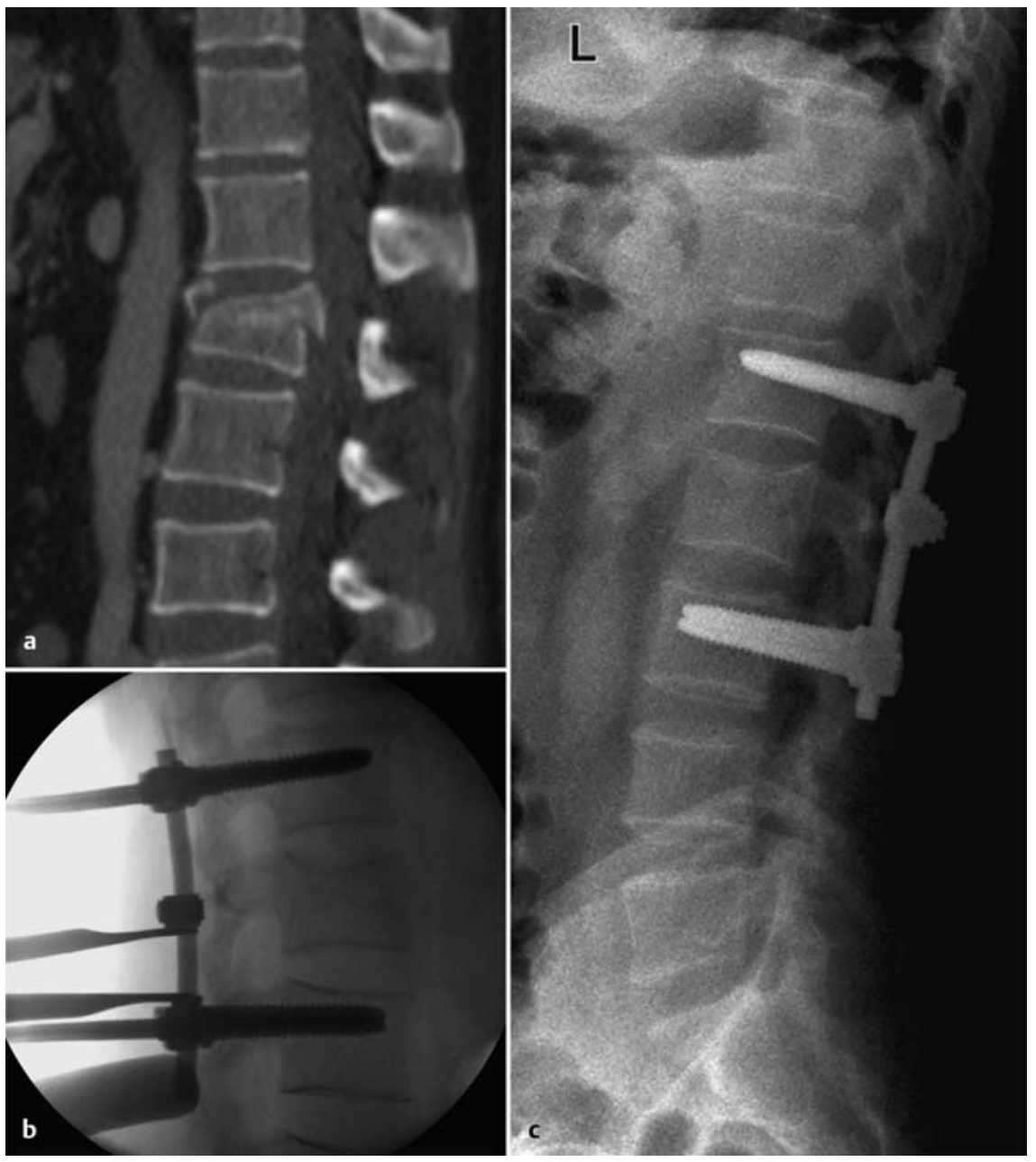

Abb. 1 a bis c Die Aufrichtung des Wirbelkörpers über Ligamentotaxis. Im präoperativen CT erkennt man das in den Spinalkanal hineinreichende Fragment der Wirbelkörperhinterkante. Durch die intraoperative Aufrichtung wird eine Reposition des Knochenfragments und eine Aufrichtung der Kyphose erreicht.

\section{Frische traumatische Frakturen von ansonsten gesunden Patienten ohne Osteoporose}

\section{OP-Indikation}

Die Stellung der OP-Indikation hängt im Wesentlichen von der erwarteten Stabilität ab. Sofern keine neurologischen Defizite vorliegen, ist die Einschätzung der biomechanischen Stabilität der frakturierten Wirbelsäule jedoch schwierig. Die Arbeitsgemeinschaft „Wirbelsäule“ der Deutschen Gesellschaft für Unfallchirurgie hat folgende morphologische Instabilitätskriterien erarbeitet [43]:

- Kontinuitätsunterbrechungen der Wirbelsäule

- Einengungen des Spinalkanals

- Abweichungen vom physiologischen Profil der Wirbelsäule: Dazu bedarf es der Messung des Grund-DeckplattenWinkels (GDW) zwischen der Deckplatte des über dem frakturierten Wir- belkörper liegenden Wirbelkörpers und der Grundplatte des frakturierten (monosegmental) bzw. bei frakturierter Grundplatte der Grundplatte des darunter liegenden Wirbelkörpers (bisegmental). Entscheidend ist bei der Messung nicht der absolute Winkel, sondern die Abweichung vom individuellen sagittalen Profil der Wirbelsäule. Daher wird die Differenz des physiologischen Krümmungswinkels und dem gemessenen GDW berechnet und als $\delta$-GDW angegeben. Bei Abweichung unter $15-20^{\circ}$ ist keine Zunahme der Abweichung auf korrekturbedürftige Werte $\mathrm{zu}$ erwarten; bei $\mathrm{Ab}$ weichungen über $15-20^{\circ}$ würde man im Verlauf einer funktionellen Therapie Abweichungen auf korrekturbedürftige Werte erwarten.

- Entscheidend ist auch die Abweichung in der frontalen Ebene. Daher wird der Skoliosewinkel gemessen. Ein Skolio- sewinkel unter $10^{\circ}$ gilt als Stabilitätszeichen.

- Grad der Zerstörung des Wirbelkörpers. Dabei werden das zerstörte Volumen und die Frakturdislokation beurteilt.

- Einengung des Spinalkanals.

- Minderung der Wirbelkörperhinterkantenhöhe.

Instabile Frakturen oder Frakturen mit drohender Instabilität stellen eine OP-Indikation dar.

\section{OP-Strategie}

Nicht nur die knöchernen Strukturen der Wirbelsäule, sondern auch die Bandscheiben und Bandstrukturen haben einen maßgeblichen Einfluss auf die Stabilität der Wirbelsäule. Aufgrund der fehlenden Rekonstruktion der vorderen Säule mit den Bandscheiben führt daher eine alleinige dorsale Spondylodese bei einigen Frakturen zu einem nicht tolerierbaren sekundären Repositionsverlust $[22,25]$. Knöcherne Defizite in der vorderen Säule können in 20\% persisieren und zu Pseudarthrosen und einem Verlust der Reposition führen [6].

Eine biomechanische Finite-ElementeAnalyse hat gezeigt, dass die Stabilität bei zusätzlicher ventraler Versorgung deutlich besser ist [31]. Daher können meist nur A1- und A2-Verletzungen ohne Instabilitätszeichen durch eine alleinige dorsale Instrumentierung therapiert werden: Tab. 3 gibt einen Überblick über die empfohlenen Behandlungsstrategien.

Bei vorhandener oder drohender Instabilität ist additiv die Aufrichtung und Stabilisierung der vorderen Säule notwendig. Dazu stehen mit der Thorakotomie und der Lumbotomie 2 Zugänge zur Verfügung, mit denen die mittlere BWS, der thorakolumbale Übergang und die LWS erreicht werden können. Der transthorakale Zugang bietet ausreichend Sicherheit, akzeptable Operationszeiten und einen akzeptablen Blutverlust [33] sowie eine insgesamt niedrige Komplikationsrate [23]. Der klassische transthorakale Zugang erfolgt über eine Thorakotomie, zur Unterstützung werden selbsthaltende Retraktorsysteme verwendet (Abb. 2).

Alternativ kann der Zugang endoskopisch über mehrere Trokare ausgeführt werden [4]. Die endoskopische Technik führt zu einer Reduktion der Morbidität 
Tab. 3 Therapieempfehlungen zur Versorgung von Verletzungen der Brust- und Lendenwirbelsäule [43].

\begin{tabular}{|c|c|c|}
\hline A & A1.1 Deckplattenimpression & frühfunktionelle Behandlung \\
\hline & A1.2 Keilbruch & $\begin{array}{l}\delta-G D W<15-20^{\circ}: \text { frühfunktionell } \\
\delta-G D W>15-20^{\circ}: \text { mindestens monosegmentale dorsale Instrumentierung }\end{array}$ \\
\hline & A1.3 Impaktionsbruch & wie $A 1.2$ \\
\hline & A2.1 sagittaler Spaltbruch & wie $A 1.2$ \\
\hline & A2.2 frontaler Spaltbruch & wie $A 1.2$ \\
\hline & A2.3 Kneifzangenbruch & $\begin{array}{l}\text { bisegmentaler } \delta-G D W<15-20^{\circ} \text { : frühfunktionell } \\
\text { bisegmentaler } \delta-G D W>15-20^{\circ} \text { : mindestens monosegmentale dorsale Instrumentierung }\end{array}$ \\
\hline & A3.1 inkompletter Berstungsbruch & $\begin{array}{l}\delta \text {-GDW }<15-20^{\circ} \text { und Skoliosewinkel }<10^{\circ} \text { : frühfunktionell } \\
\delta \text {-GDW }>15-20^{\circ} \text { und Skoliosewinkel }>10^{\circ} \text { : mindestens monosegmentale dorsale Instru- } \\
\text { mentierung, monosegmentale dorsale Sponylodese erwägen } \\
\text { Zerstörung des } W K<1 / 3 \text { : ventrale Fusion optional } \\
\text { Zerstörung des } W K>1 / 3 \text { : monosegmentale ventrale Rekonstruktion }\end{array}$ \\
\hline & A3.2 Berstungsspaltbruch & $\begin{array}{l}\text { ohne Dislokation: wie A3.1 } \\
\text { mit Dislokation: wie A3.3 }\end{array}$ \\
\hline & A3.3 kompletter Berstungsbruch & $\begin{array}{l}\delta \text {-GDW }<15-20^{\circ} \text { und Skoliosewinkel }<10^{\circ} \text { : frühfunktionell } \\
\delta \text {-GDW }>15-20^{\circ} \text { und Skoliosewinkel }>10^{\circ} \text { : } \\
\text { Je größer die Fragmente und je stärker die Einengung des Spinalkanls, desto eher ist die } \\
\text { OP-Indikation gegeben. Mindestens bisegmental. Dorsale Instrumentierung, mono- oder } \\
\text { bisegmentale dorsale Spondylodese erwägen. } \\
\text { Bei guter Reposition und Knochenqualität auch allein ventrale Stabilisierung möglich. }\end{array}$ \\
\hline B & $\begin{array}{l}\text { B1.1 transligamentäre Flexionsdistraktions- } \\
\text { verletzung mit Diskuszerreißung }\end{array}$ & $\begin{array}{l}\text { Reposition, dorsale Instrumentierung mit Spondylodese, monosegmentale ventrale } \\
\text { Rekonstruktion ist optional }\end{array}$ \\
\hline & $\begin{array}{l}\text { B1.2 transligamentäre Flexionsdistraktions- } \\
\text { verletzung mit Korpusfraktur }\end{array}$ & $\begin{array}{l}\text { Reposition, dorsale Instrumentierung mit Spondylodese, monosegmentale ventrale } \\
\text { Rekonstruktion bei } \delta \text {-GDW }>15-20^{\circ}\end{array}$ \\
\hline & $\begin{array}{l}\text { B2.1 horizontale Wirbelzerreißung } \\
\text { (Chance-Fraktur) }\end{array}$ & Reposition und bisegmentale dorsale Instrumentierung \\
\hline & $\begin{array}{l}\text { B2.2 Flexionsspondylolyse mit } \\
\text { Bandscheibenzerreißung }\end{array}$ & $\begin{array}{l}\text { Reposition und mindestens monosegmentale dorsale Instrumentierung mit Spondylo- } \\
\text { dese, monosegmentale ventrale Rekonstruktion ist optional }\end{array}$ \\
\hline & B2.3 Flexionsspondylolyse mit Korpusfraktur & wie B1.2 \\
\hline & B3.1 Hyperextensionssubluxation & $\begin{array}{l}\text { Reposition und mindestens dorsale Instrumentierung, monosegmentale ventrale Rekon- } \\
\text { struktion ist optional }\end{array}$ \\
\hline & B3.2 Hyperextensionsspondylolyse & wie B3.1 \\
\hline & B3.3 hintere Luxation & wie B3.1 \\
\hline C & C1.1 Rotationskeilbruch & $\begin{array}{l}\text { Reposition und mindestens dorsale Instrumentierung mit Querverbinder und monoseg- } \\
\text { mentaler Spondylodese, monosegmentale ventrale Rekonstruktion bei } \delta-G D W>15-20^{\circ}\end{array}$ \\
\hline & C1.2 Rotationsspaltbruch & $\begin{array}{l}\text { Reposition und mindestens bisegmentale dorsale Instrumentierung mit Querverbinder } \\
\text { und Spondylodese, monosegmentale ventrale Rekonstruktion bei } \delta-G D W>15-20^{\circ}\end{array}$ \\
\hline & C1.3 Rotationsberstungsbruch & wie $\mathrm{C} 1.2$ \\
\hline & C2.1 Rotation mit B1-Verletzung & wie $\mathrm{C} 1.2$ \\
\hline & C2.2 Rotation mit B2-Verletzung & wie $C 1.2$ \\
\hline & C2.3 Rotation mit B3-Verletzung & wie $C 1.2$ \\
\hline & C3.1 Slice-Fraktur & Reposition und mindestens bisegmentale dorsale Instrumentierung mit Querverbinder \\
\hline & C3.2 Rotationsschrägbruch & $\begin{array}{l}\text { Reposition und mehrsegmentale dorsale Instrumentierung mit Querverbinder, monoseg- } \\
\text { mentale ventrale Rekonstruktion bei } \delta-G D W>15-20^{\circ}\end{array}$ \\
\hline
\end{tabular}

[29] sowie zu einer Reduktion der postoperativen Schmerzen und zu einer verkürzten Hospitalisierung [8]. Während bei Einführung der ventralen Zugänge noch ein Viszeral- oder Thoraxchirurg für den Zugang hinzugezogen wurde, ist nach neueren Untersuchungen die Konsultation eines „Zugangschirurgen“ nicht notwendig. Bei ausreichend trainierten Wirbelsäulenchirurgen zeigten sich keine höheren Komplikationsraten [20].
Bezüglich des zeitlichen Ablaufs kann die dorsoventrale Fusion 2-zeitig oder simultan durchgeführt werden. Die simultane Operation ist sicher und effektiv [32]. Es konnten eine niedrigere Pseudarthroserate, eine bessere Korrektur der 


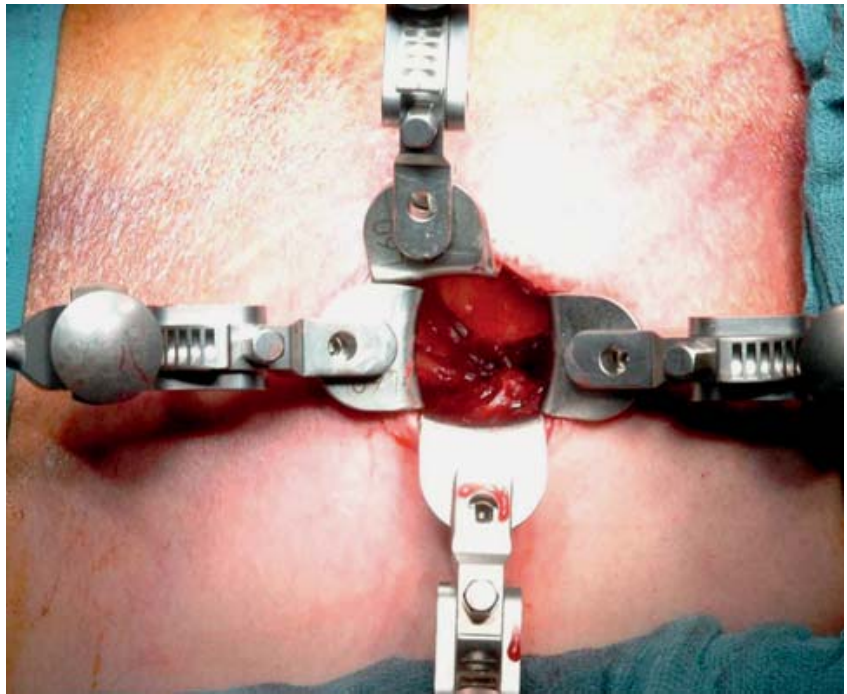

Abb. 2 Minimalinvasiver transthorakaler Zugang unter Verwendung eines Selbsthaltesystems (Synframe, Fa. Synthes, Umkirch).

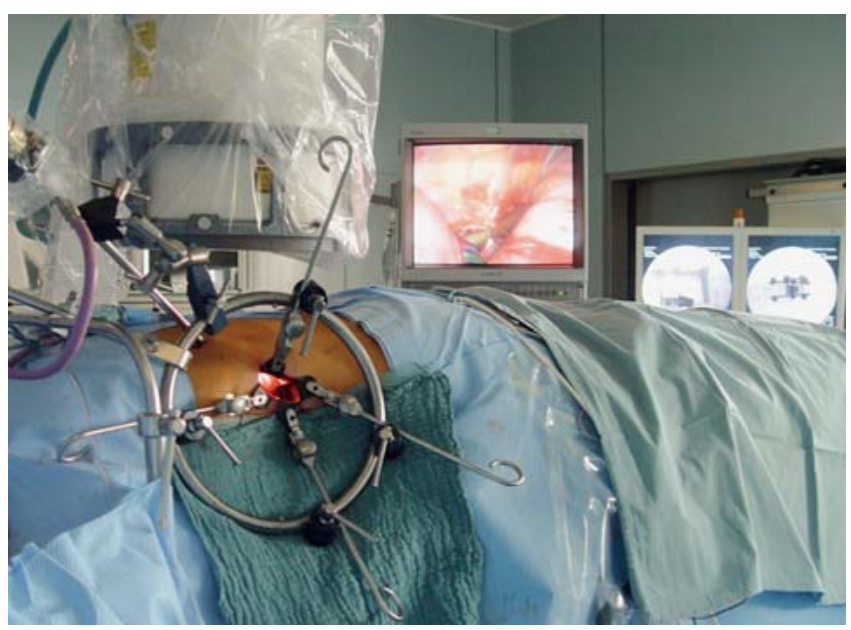

Abb. 3 Lagerung des Patienten und Aufbau der notwendigen technischen Geräte im OP-Saal bei einer transthorakalen ventralen Stabilisierung. Dabei ist zu beachten, dass der Operateur das Endoskopiebild, die Flouroskopiemonitore, den Monitor des Navigationsgeräts und idealerweise die wichtigsten Kreislaufparameter im Blick hat.

Deformität, eine niedrigere Morbidität [12], eine insgesamt kürzere Operationszeit, ein geringerer Blutverlust, eine kürzere Hospitalisierung und insgesamt weniger Komplikationen [37] nachgewiesen werden. Allerdings stellt das Vorgehen höhere Anforderungen an das behandelnde Zentrum bez. Logistik, Lagerung und personeller Ausstattung. An unserer Klinik wird, wenn möglich, das simultane Vorgehen bevorzugt (Abb. 3).

Die simultan durchgeführte dorsoventrale Stabilisierung hat sich als vorteilhaft erwiesen.

Uneinigkeit herrscht bislang in der Frage, ob eine suffiziente Stabilisierung der ventralen Säule auch über einen alleinigen dorsalen Zugang möglich ist. Einerseits berichten einige aktuelle Studien von einem suffizienten Aufbau der vorderen Säule mit adäquater Korrektur der Kyphose durch die Implantation eines PLIF oder die transpedikuläre Implanta- tion von autologem oder allogenem Knochenmaterial $[36,43]$. Andererseits zeigt die groß angelegte Multicenterstudie MCSII der Deutschen Gesellschaft für Unfallchirurgie (DGU), dass ein kombinierter Zugang die posttraumatische Deformität am besten wiederhergestellt hat und der verbleibende Kyphosewinkel signifikant geringer ist [34]. Die Autoren berichten jedoch andererseits von einem besseren klinischen und funktionellen Outcome nach alleiniger dorsaler Versorgung. Diese Aussage ist jedoch fraglich und wahrscheinlich bedingt durch mehr B- und C-Verletzungen in der Gruppe der dorsoventralen Versorgung. Zur abschließenden Klärung der Frage bedarf es weiterer prospektiver multizentrischer Studien.

\section{Dorsale perkutane Operationsverfahren}

Für die dorsale Instrumentierung stehen verschiedene perkutane Verfahren zur Verfügung, welche sich in der klinischen
Anwendung in unserem Zentrum bewährt haben. Die Vorteile der perkutanen Technik sind eine schnellere Mobilisierung, eine kürzere Hospitalisierung und ein geringerer Blutverlust. Als Nachteil und indikationslimitierend kann die fehlende Möglichkeit der knöchernen Dekompression des Spinalkanals gewertet werden.

Perkutane Verfahren zur dorsalen Stabilisierung werden zunehmend klinisch etabliert.

\section{Zementaugmentation der Pedikelschrauben}

Sowohl bei der perkutanen als auch bei der offenen dorsalen Instrumentierung besteht die Möglichkeit der Zementaugmentation der Pedikelschrauben. Zur Anwendung kommt dabei meistens Polymethylmethacrylat (PMMA). Studien konnten bei verminderter Knochendichte eine höhere Stabilität von zementaugmentierten Schrauben nachweisen [47]. Bei jungen knochengesunden Patienten wird eine Zementaugmentation hingegen nicht empfohlen [43] (Tab. 3).

\section{Osteoporotische Frakturen}

Die operative Therapie der osteoporotischen Wirbelkörperfraktur des alten Menschen unterscheidet sich maßgeblich von den vorangegangenen Therapieprinzipien. Bei der Indikationsstellung muss aufgrund des höheren Lebensalters und oft vorhandener Begleiterkrankungen mit einem höheren Narkoserisiko gerechnet werden.

Die operative Therapie der osteoporotischen Wirbelkörperfraktur des alten Menschen unterscheidet sich maßgeblich von der operativen Therapie von Wirbelsäulenverletzungen bei jungen knochengesunden Patienten.

Die häufigsten osteoporotischen Frakturen sind A1.3-Frakturen, welche bei fehlenden Instabilitätszeichen meist frühfunktionell konservativ behandelt werden können. Dabei stehen beim alten Menschen die forcierte Mobilisierung zur Vermeidung von immobilisationsbedingten Komplikationen sowie eine adäquate Schmerztherapie im Vordergrund. Außerdem sollten - sofern noch nicht geschehen - eine Bestimmung der Knochendichte durchgeführt und eine stadiengerechte medikamentöse Osteoporosetherapie eingeleitet werden. 
Eine Indikation zur operativen Versorgung besteht bei therapieresistenten Schmerzen. Sofern die Wirbelkörperhinterkante nicht disloziert ist, eignet sich eine minimalinvasive transpedikuläre Aufrichtung des Wirbelkörpers mittels eines eingebrachten Ballonkatheters und Fixierung des Repositionsergebnisses mit Knochenzement (Kyphoplastie) oder eine alleinige Stabilisierung mit Knochenzement (Vertebroplastie). Studien haben gezeigt, dass bei persistierenden Schmerzen eine Kyphoplastie in Bezug auf Schmerzreduktion und Lebensqualität der konservativen Therapie überlegen [44] und ebenbürtig zur Vertebroplastie ist. Bei der Vertebroplastie zeigten sich dabei weniger Zementleckagen [38].

Eine Weiterentwickelung besteht in der Implantation von Titannetzen. Diese bedingt eine bessere Beibehaltung der Wirbelkörperhöhe bei gleicher biomechanischer Stabilität und geringerem Zementverbrauch [41].

Bei dislozierter Hinterkante, Instabilitätszeichen oder progredientem Kyphosewinkel ist auch bei osteoporotischen Frakturen eine dorsale Instrumentierung indiziert. Dabei sollten präoperativ eine Knochendichtemessung erfolgen und die Schrauben bei gesicherter Osteoporose zementaugmentiert implantiert werden. Zusätzlich zur dorsalen Instrumentierung kann eine Kyphoplastie oder Vertebroplastie des frakturierten Wirbelkörpers zur Stabilisierung der vorderen Säule erfolgen. Perkutane Techniken sind beim älteren Patienten vorzuziehen.

\section{OP-Vorbereitung und intraoperative Kontrolle}

\section{Anforderungen an die behandelnde Klinik}

Für die konservative und operative Behandlung von Wirbelkörperfrakturen müssen jederzeit die optimalen technischen und personellen Voraussetzungen gegeben sein. Patienten, welche frühfunktionell konservativ behandelt werden, müssen nicht zwingend in ein Wirbelsäulenzentrum verlegt werden [2]. Retrospektive Auswertungen zeigten eine ausreichend hohe Therapiequalität auch ohne die Konsultation eines spezialisierten Wirbelsäulenchirurgen. Allerdings sollte in der behandelnden Klinik ein Mindestmaß an diagnostischer Ausstattung vorhanden sein, dazu zählen ein CT und ein MRT.
Bei morphologischen Zeichen der Instabilität oder dem Auftreten von neurologischen Defiziten wird schnellstmöglich die Verlegung in ein spezialisiertes Zentrum empfohlen. Aufgrund des hohen logistischen Aufwands und der Notwendigkeit der Spezialisierung ist die operative Behandlung an Wirbelsäulenzentren gebunden. Diese müssen ventrale Versorgungen und sämtliche Komplikations- und Revisionsoperationen jederzeit vorhalten können.

Der Transport muss dabei so schonend wie möglich gestaltet werden, eine Stabilisierung des Patienten mit geeigneten Rettungsmitteln (z.B. Vakuummatratze) ist anzustreben, außerdem ist die Möglichkeit eines Hubschraubertransports zu prüfen.

Bei morphologischen Zeichen der Instabilität oder dem Auftreten von neurologischen Defiziten wird schnellstmöglich die Verlegung in ein spezialisiertes Wirbelsäulenzentrum empfohlen.

\section{Zeitliche Planung}

Kriterien für eine dringliche oder notfallmäßige Versorgung von Frakturen der Brust- und Lendenwirbelsäule stellen neurologische Defizite, Zeichen der Instabilität und Fehlstellungen dar. In diesem Fall sollte, wie oben beschrieben, frühzeitig Kontakt mit einem Wirbelsäulenzentrum aufgenommen und ggf. die Übernahme des Patienten organisiert werden.

In der spezialisierten Klinik erfolgt bei folgenden Kriterien eine sofortige Operation:

- Instabilität, drohendes neurologisches Defizit

- neurologisches Defizit mit relevanter Spinalkanaleinengung, bei analgosedierten Patienten auch, wenn das neurologische Defizit aufgrund der Bildgebung wahrscheinlich ist

- offene Wirbelsäulenverletzung

- grobe Dislokation

- hochgradig instabile Wirbelkörperfrakturen im Rahmen der Behandlung polytraumatisierter oder mehrfachverletzter Patienten

Auch wenn keine Notfallindikation vorliegt, wird eine zeitnahe operative Therapie angestrebt. Ziel ist dabei eine Versorgung innerhalb von 72 Stunden nach Aufnahme. Dies senkt nachgewiesenermaßen die Morbidität und Mortalität [5]. Das Risiko der postoperativen Wund- infektion steigt bei langem präoperativem Krankenhausaufenthalt [14], nach 1 Woche ist zudem die Reposition erheblich erschwert [9]. In unserer Klinik werden $80-90 \%$ der Patienten mit frischen Wirbelkörperfrakturen am Aufnahmetag operativ versorgt.

\section{Aufklärung}

Gemäß den gesetzlichen Vorgaben muss eine ausführliche Aufklärung unter Beachtung von alternativen Therapiemöglichkeiten und Komplikationen erfolgen. Dabei wird ein schriftliches Gesprächsprotokoll verfasst und vom Arzt und, sofern möglich, vom Patienten unterschrieben. Folgende Komplikationen sollten erwähnt werden:

- Gefäß-, Nervenverletzungen

- postoperative Wundinfektionen

- Thrombose

- Verletzung von Myelon und Nervenwurzel, neurologische Defizite

- Duraverletzungen, Liquorfisteln

- Nachsinterung

- Anschlussfraktur

- Anschlussdegeneration

- Repositionsverluste, Materialdislokation

- zugangsbedingte Komplikationen (Leber-/Milz-/Nierenrupturen, Darmperforation, Pneumothorax, Lungenperforation, Verletzung der Aorta)

- Lagerungsschäden

\section{Anästhesie}

Jüngere Patienten nach Sturz oder Hochrasanztraumen haben oft Begleitverletzungen, nicht selten Thorax- oder Schädel-Hirn-Verletzungen, welche das Narkoserisiko erhöhen. Alte Menschen mit osteoporotischen Frakturen leiden oft unter internistischen Begleiterkrankungen. Die Narkoseführung ist aufgrund dieser Tatsachen und der möglichen Thorakotomie sowie der meistens verwendeten Bauchlagerung anspruchsvoll. Eine enge Zusammenarbeit zwischen Chirurg und Anästhesist sowie eine strenge Indikationsstellung helfen, die operationsassoziierte Morbidität und Mortalität zu senken. Die Morbiditätsund Mortalitätsrate bei Wirbelsäulenverletzungen korreliert eng mit der ASA-Klasse (American Society of Anesthesiology) [13].

Neben den allgemein üblichen Narkosevorbereitungen sollte auf die Bereitstellung von Blutkonserven, eine ausreichende Überwachungsmöglichkeit aufgrund der Bauchlagerung und bei ventralen Fu- 
sionen auf die Verwendung von Doppellumentuben geachtet werden. Eine intraoperative Antibiose ist bei den meisten Wirbelsäuleneingriffen indiziert. Postoperativ muss eine geeignete Überwachung des Patienten gewährleistet sein.

Die Narkoseführung bei wirbelsäulenverletzten Patienten ist anspruchsvoll, eine enge Zusammenarbeit zwischen Chirurg und Anästhesist ist unerlässlich.

\section{Vorbereitung der Bilder}

Die präoperativ angefertigten Röntgen-, CT- und MRT-Aufnahmen sollten dem Operateur intraoperativ zur Verfügung stehen. Dazu eignet sich am besten ein PACS-System mit der Möglichkeit des berührungslosen Blätterns. Alternativ können im Operationssaal gedruckte Bilder aller relevanten Schichten aufgehängt werden. In den Bildern werden idealerweise die Pedikelbreite sowie die Tiefe und Höhe der Wirbelkörper präoperativ vermessen, um geeignetes Osteosynthesematerial zu wählen.

\section{Materialplanung}

Sowohl für die dorsale Instrumentierung als auch für den ventralen Wirbelkörperersatz steht eine Vielzahl kommerziell verfügbarer Implantate zur Auswahl. Schrauben und Querträger werden fast ausnahmslos aus Titan gefertigt und verfügen über sehr gute Materialeigenschaften hinsichtlich ihrer Bruchfestigkeit und Verträglichkeit.

Beim ventralen Wirbelkörperersatz herrscht noch Uneinigkeit, ob die Verwendung von Titancages oder die Implantation von allogenem oder autologem Knochengewebe vorteilhafter ist. Fibular Grafts haben die gleiche Steifheit wie Titancages [10], aber bei Cages zeigt sich im Vergleich zum Knochenspan ein besseres sagittales Alignement [34]. An unserem Zentrum werden meist Titancages verwendet.

\section{Navigation}

Bedingt durch die technischen Entwicklungen der vergangenen Jahre stieg der Stellenwert der intraoperativen Navigation stetig. Mit der aktuellen Gerätegeneration stehen einfach $\mathrm{zu}$ bedienende alltagstaugliche Möglichkeiten der 3-dimensionalen intraoperativen Bildgebung zur Verfügung.

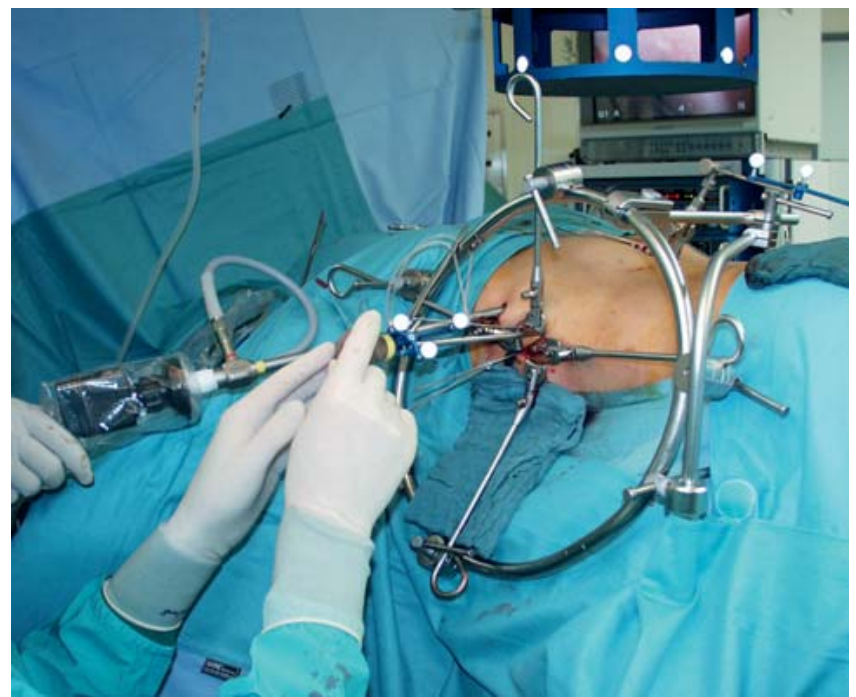

Abb. 4 Verwendung von navigationsfähigen OP-Instrumenten am Beispiel einer minimalinvasiven simultanen dorsoventralen Stabilisierung. Am Instrument, am C-Bogen und an der am Dornfortsatz angebrachten Referenzierung sind Reflexionskugeln angebracht.

Die Wirbelsäulenchirurgie eignet sich aufgrund von teilweise nur eingeschränkt sichtbaren anatomischen Verhältnissen und der Nähe zu wichtigen Organstrukturen (Rückenmark, Aorta) besonders für eine Navigation. Außerdem ist insbesondere bei der verletzten Wirbelsäule die Orientierung aufgrund veränderter anatomischer Landmarken eingeschränkt.

Ein aktuelles Review bemängelte, dass die bislang publizierten Studien meistens aufgrund der Größe des Patientenkollektivs und des retrospektiven Studiendesigns eine limitierte Aussagekraft haben [39]. In den letzten 2 Jahren wurden jedoch zunehmend auch prospektive kontrollierte Studien publiziert, sodass von einer steigenden Evidenz in den nächsten Jahren auszugehen ist.

Insgesamt zeigten die bislang publizierten Studien, dass die Navigation technisch möglich ist. Es gibt Studien, die eine verbesserte Präzision der Implantatlage zeigten [35], andere konnten keine Unterschiede hinsichtlich der Implantatlage zeigen [7]. Die Verwendung von 3-D-Navigation kann die Strahlendosis (effektive Dosis) gegenüber der konventionellen Fluoroskopie verringern [24].

Eine Studie unserer Arbeitsgruppe konnte bei der navigierten ventralen Fusion Anzeichen für eine bessere Schonung der angrenzenden intakten Endplatte finden [7]. Allerdings verlängert sich die Operationszeit durch die Verwendung der Navigation signifikant.

In der Entwicklungsphase ist das System der navigierten Power Control, welches bei der Laminektomie die Fräse bei einem zu geringen Abstand zur Dura stoppt [40]. In der Hals-Nasen-OhrenHeilkunde hat ein solches System bereits den Einzug in die tägliche Praxis gefunden.

Zusätzlich zur Navigation ist durch die intraoperative 3-D-Bildgebung eine Kontrolle der Schraubenlage möglich. Vor Einführung der Technik wurde die Schraubenlage im postoperativen CT kontrolliert. Dokumentierte Schraubenfehllagen bedürfen dabei eines operativen Korrektureingriffs mit erneutem Mortalitäts- und Morbiditätsrisiko. Die Anwendung einer intraoperativen Kontrolle konnte eine Verringerung der sekundären Revisionsrate um $0,5 \%$ bei einer intraoperativen primären Revisionsrate von $2,7 \%$ zeigen [3].

Technische Voraussetzungen für die Durchführung einer Navigation sind neben einem 3-D-Bildwandler und einem Navigationssystem auch OP-Instrumente, welche vom Navigationssystem erkannt werden, sowie ein strahlendurchlässiger Operationstisch, welcher meist aus Karbon gefertigt wird.

Zusammenfassend kann zum jetzigen Zeitpunkt gesagt werden, dass die Navigation eine wertvolle Ergänzung ist, die aber mit hohen Kosten verbunden ist. Derzeit muss noch eine Lernkurve (v.a. bez. der Operationszeit) durchschritten werden (Abb. 4).

Navigationssysteme können die Patientensicherheit erhöhen. 


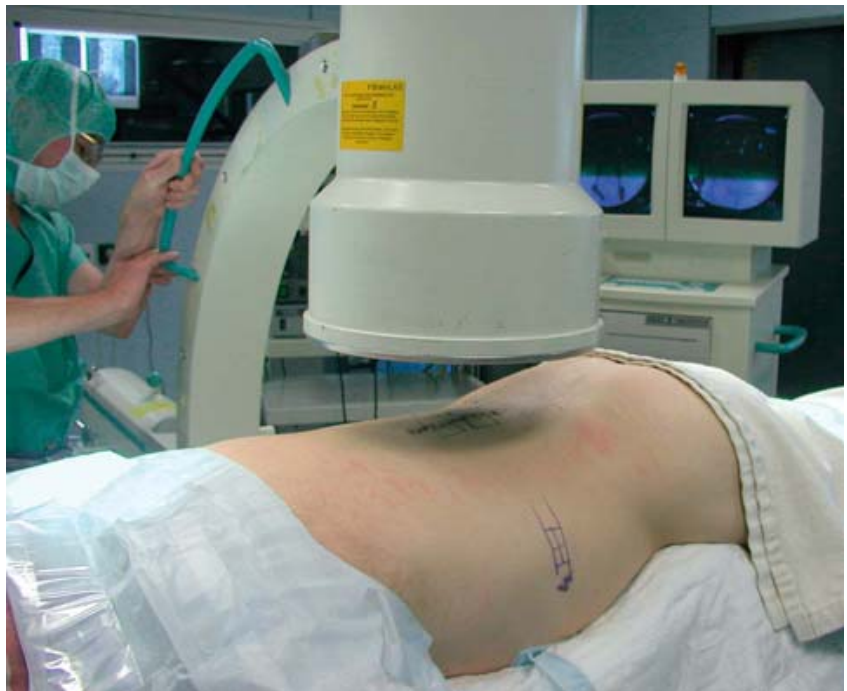

Abb. 5 Hyperlordosierende Lagerung und Röntgenkontrolle des Repositionsergebnisses im OP-Saal.

\section{Neuromonitoring}

Ein Neuromonitoring hält zunehmend Einzug in die Wirbelsäulenchirurgie. Die intraoperative Ableitung von motorisch und sensibel evozierten Potenzialen kann die OP-Sicherheit erhöhen [1]. Allerdings konnte in einer aktuellen Studie keine signifikante Verbesserung des postoperativen neurologischen Status gezeigt werden. Dazu sind größer angelegte prospektive Studien notwendig [11]. Die Bereitstellung des intraoperativen Neuromonitorings ist an einen erheblichen technischen und personellen Aufwand gebunden. Ob sich die Technik in der klinischen Routine durchsetzt, ist noch nicht absehbar.

\section{Lagerung und geschlossene Reposition}

Die Lagerung für die dorsale Instrumentierung erfolgt in Bauchlagerung im leichten Durchhang (Hyperlordose). Diese wird durch entsprechende Unterpolsterung von Thorax und Becken durch Lagerungskissen erreicht. Die lordosierende Lagerung führt bei Frakturen der unteren BWS und LWS häufig bereits zu einer teilweisen Wiederaufrichtung des gebrochenen Wirbelkörpers [30] und sogar zu einer Reposition der Hinterkante. Die geschlossene Reposition durch die Lagerung wird idealerweise röntgenologisch kontrolliert. Dabei können die zu operierenden Wirbelkörper und der geplante Hautschnitt angezeichnet werden (Abb. 5).

Die Arme werden bei Frakturen der mittleren BWS und LWS ausgelagert, dabei sollte eine Elevation im Schultergelenk über $90^{\circ}$ vermieden werden. Bei Fraktu- ren der oberen BWS empfiehlt sich eine Anlagerung der Arme.

Nach Möglichkeit sollte auch bei nicht navigationsgestützten Eingriffen ein röntgenstrahlendurchlässiger Karbontisch für eine Verbesserung der intraoperativen Bildqualität zum Einsatz kommen.

Bei der ventralen Fusion bevorzugen wir ebenfalls eine Bauchlagerung. Die Vorteile bestehen in der Möglichkeit einer simultanen dorsalen Instrumentierung und erleichtertern Repositionsmöglichkeiten. Es kommt zu einem besseren Spontanalignement der Wirbelsäule und die Gefahr der Fixierung in einer skoliotischen Fehlstellung, wie sie in Seitenlage auftreten kann, ist minimiert. In Bauchlage sind die Wirbelkörper von BWK4 bis LWK5 von ventral erreichbar.

Bei der Lagerung muss auf eine ausreichende Polsterung der Extremitäten sowie auf eine suffiziente Fixierung des Patienten auf dem OP-Tisch mithilfe von Stützen und Gurtsystemen geachtet werden. Die Lagerung findet in enger Kooperation mit den Kollegen der Anästhesie statt.

\section{Literatur}

${ }^{1}$ Alemo S, Sayadipour A. Role of intraoperative neurophysiologic monitoring in lumbosacral spine fusion and instrumentation: a retrospective study. World Neurosurg 2010; 73: 72-76

${ }^{2}$ Baldwin KM, Ryb GE, Miller D et al. Is spine consultation needed for all thoracolumbar fractures? Evaluation of a subspecialist-sparing protocol for screening and management of stable fractures. J Trauma 2010; 69: 14911495

3 Beck M, Mittlmeier T, Gierer P et al. Benefit and accuracy of intraoperative $3 \mathrm{D}$-imaging after pedicle screw placement: a prospective study in stabilizing thoracolumbar fractures. Eur Spine J 2009; 18: 1469-1477

${ }^{4}$ Beisse $R$. Endoscopic surgery on the thoracolumbar junction of the spine. Eur Spine J 2010; 19 (Suppl. 1): S52-S65

5 Bellabarba C, Fisher C, Chapman JR et al. Does early fracture fixation of the thoracolumbar spine fractures decrease morbidity or mortality? Spine 2010; 35 (Suppl. 9): S138-S145

6 Berven S, Kao H, Deviren V et al. Treatment of thoracic pseudarthosis in the adult: is combined surgery necessary? Clin Orthop Relat Res 2003; 411: 25-31

7 Blattert TR, Springwald J, Glasmacher S et al. Navigationsgestützte Rekonstruktion der vorderen Säule bei Verletzungen im Brustwirbel- und thorakolumbalen Übergangsbereich. Unfallchirurg 2008; 111: 878-885

8 Bühren V, Beisse R, Potulski M. Minimal-invasive ventrale Spondylodesen bei Verletzungen der Brust- und Lendenwirbelsäule. Chirurg 1997; 68: 1076-1084

${ }^{9}$ Bühren V. Verletzungen der Brust- und Lendenwirbelsäule. Chirurg 2001; 72: 865-879

10 Cardenas RJ, Javalkar V, Patil S et al. Comparison of allograft bone and titanium cages for vertebral body replacement in the thoracolumbar spine: a biomechanical study. Neurosurgery 2010; 66 (6 Suppl. Operative): 314318

11 Castellon AT, Meves R, Avanzi O. Intraoperative neurophysiologic spinal cord monitoring in thoracolumbar burst fractures. Spine 2009; 34: 2662-2668

12 Dick J, Boachie-Adjei O, Wilson M. One-stage versus two-stage anterior and posterior spinal reconstruction in adults. Spine 1992; 17 : S310-S316

${ }^{13}$ Fu KG, Smith JS, Polly DW et al. Correlation of higher preoperative American Society of Anesthesiology grade and increased morbidity and mortality rates in patients undergoing spine surgery. J Neurosurg Spine 2011; 13: $1-5$

14 Geffers C, Gastmeier P, Daschner F et al. Prävention postoperativer Wundinfektionen. Zentralbl Chir 2001; 126: 84-92

15 Glaesener JJ, Hasse W, Exner G et al. Thoracopulmonary complications of fresh fractures of the thoracic spine with neurologic damage. Unfallchirurgie 1992; 18: 274-279

16 Gonschorek O, Hauck S, Bühren V. Therapiestandards bei Verletzungen der BWS/LWS. Trauma Berufskrankh 2009; 11: 117-123

17 Hauser CJ, Visvikis G, Hinrichs C et al. Prospective validation of computed tomographic screening of the thoracolumbar spine in trauma. J Trauma 2003; 55: 228-235

18 Inaba K, DuBose JJ, Barmparas G et al. Clinical examination is insufficient to rule out thoracolumbar spine injuries. J Trauma 2001; 70: 174-179

${ }^{19}$ Jansson K, Blomqvist P, Svedmark P et al. Thoracolumbar vertebral fractures in Sweden: an analysis of 13, 496 patients admitted to hospital. Eur J Epidemiol 2010; 25: 431-437

20 Jarrett CD, Heller JG, Tsai L. Anterior exposure of the lumbar spine with and without an "access surgeon": morbidity analysis of 265 consecutive cases. J Spinal Disord Tech 2009; 22: 559-564

21 Josten C, Katscher S. Radiologische Diagnostik bei Wirbelsäulenverletzungen. Akt Traumatol 2003; 33: 157-164

22 Josten C, Katscher S, Gonschorek O. Therapiekonzepte bei Frakturen des thorakolumbalen Übergangs und der Lendenwirbelsäule. Orthopäde 2005; 34: 1021-1032

${ }^{23}$ Kim NR, Hong SH, Choi JY et al. Spreading epidural hematoma and deep subcutaneous 
edema: indirect MRI signs of posterior ligamentous complex injury in thoracolumbar burst fractures. Skeletal Radiol 2010; 39: 767-772

${ }^{24}$ Kraus MD, Krischak G, Keppler P et al. Can computer-assisted surgery reduce the effective dose for spinal fusion and sacroiliac screw insertion? Clin Orthop Relat Res 2010; 468: 2419-2429

${ }^{25}$ Lakshamanan P, Jones A, Mehta J. Recurrence of kyphosis and its functional implications after surgical stabilization of dorsolumbar unstable burst fractures. Spine J 2009; 9: 1003-1009

${ }^{26}$ Looby S, Flanders A. Spine trauma. Radiol Clin North Am 2011; 49: 129-163

${ }^{27}$ Magerl F, Aebi M, Gertzbein SD et al. A comprehensive classification of thoracic and lumbar injuries. Eur Spine J 1994; 3: 184-201

${ }^{28}$ Mancini DJ, Burchard KW, Pekala JS. Optimal thoracic and lumbar spine imaging for trauma: are thoracic and lumbar spine reformats always indicated? J Trauma 2010; 69: 119 121

${ }^{29}$ McAfee PC, Regan JR, Fedder IL. Anterior thoracic corpectomy for spinal cord decompression performed endoscopically. Surg Laparosc Endosc 1995; 5: 339-348

${ }^{30}$ Müller CW, Gösling T, Mameghani A et al. Osteoporosebedingte Wirbelkörperfrakturen. Orthopäde 2010; 39: 417-424

31 Park WM, Park Y, Kim K et al. Biomechanical comparison of instrumentation techniques in treatment of thoracolumbar burst fractures: a finite element analysis. J Orthop Sci 2009; 14: 443-449

32 Peeling L, Frangou E, Hentschel S et al. Refinements to the simultaneous anterior-posterior approach to the thoracolumbar spine. J Neurosurg Spine 2010; 12: 456-461

33 Ragel BT, Kan P, Schmidt MH. Blood transfusions after thoracoscopic anterior thoracolumbar vertebrectomy. Acta Neurochir 2010; 152: 597-603

34 Reinhold M, Knop C, Beisse R et al. Operative treatment of 733 patients with acute thoracolumbar spinal injuries: comprehensive re- sults from the second, prospective, internetbased multicenter study of the Spine Study Group of the German Association of Trauma Surgery. Eur Spine J 2010; 19: 1657-1676

35 Schleicher P, Scholz M, Schnake K et al. Navigation bei thorakolumbalen Wirbelsäulenverletzungen. Trauma Berufskrankh 2009; 11: 100-105

${ }^{36}$ Schmid R, Krappinger D, Seykora P et al. PLIF in thoracolumbar trauma: technique and radiological results. Eur Spine J 2010; 19: 10791086

37 Shufflebarger HL, Grimm JO, Bui V et al. Anterior and posterior spinal fusion. Staged versus same-day surgery. Spine 1991; 16: 930933

38 Taylor RS, Fritzell P, Taylor RJ. Balloon kyphoplasty in the management of vertebral compression fractures: an updated systematic review and meta-analysis. Eur Spine J 2007; 16: 1085-1100

39 Tjardes T, Shafizadeh S, Rixen D et al. Imageguided spine surgery: state of the art and future directions. Eur Spine J 2010; 19: 25-45

40 Trantakis C, Dengl M, Grunert R et al. Evaluation of a navigated and power controlled milling system "navigated control spine" for spinal surgery. Int J Comput Assist Radiol Surg 2009; 4: 487-495

${ }^{41}$ Upasani VV, Robertson C, Lee D et al. Biomechanical comparison of kyphoplasty versus a titanium mesh implant with cement for stabilization of vertebral compression fractures. Spine 2010; 35: 1783-1788

42 Vaccaro AR, Rihn JA, Saravanja D et al. Injury of the posterior ligamentous complex of the thoracolumbar spine: a prospective evaluation of the diagnostic accuracy of magnetic resonance imaging. Spine 2009; 34: E841E847

43 Verheyden AP, Hölzl A, Ekkerlein H et al. Therapieempfehlungen zur Versorgung von Verletzungen der Brust- und Lendenwirbelsäule. Unfallchirurg 2011; 114: 9-16

${ }^{44}$ Wardlaw D, Cummings SR, van Meirhaeghe J et al. Efficacy and safety of balloon kyphoplasty compared with non-surgical care for verte- bral compression fracture (FREE): a randomised controlled trial. Lancet 2009; 373: 1016 1024

45 Winkler D, Blattert TR, Meixensberger J. Das Wirbelsäulentrauma. Notfallmedizin up2date 2007; 2: 73-89

${ }^{46}$ Wolfmann A, Bühren V. Schockraummanagement bei Verletzungen der Wirbelsäule im Rahmen eines Polytraumas. Unfallchirurg 2004; 107: 911-918

47 Zhuang XM, Yu BS, Zheng ZM et al. Effect of the degree of osteoporosis on the biomechanical anchoring strength of the sacral pedicle screws: an in vitro comparison between unaugmented bicortical screws and polymethylmethacrylate augmented unicortical screws. Spine 2010; 35: E925-E931

48 Whitesides TE. Traumatic kyphosis of the thoracolumbar spine. Clin Orthop Relat Res 1977; $128: 78-92$

49 Denis F. The three column spine and its significance in the classification of acute thoracolumbar spinal injuries. Spine 1983; 8: 817831

Dr. med. Jens Adermann

Assistenzarzt

Dr. med. Christian Schmidt

Oberarzt

Prof. Dr. med. Christoph Josten

Ärztlicher Direktor

Klinik und Poliklinik für

Unfall-, Wiederherstellungs-

und Plastische Chirurgie

Wirbelsäulenzentrum

Universitätsklinikum Leipzig

Liebigstraße 20

04103 Leipzig

jens.adermann@medizin.uni-leipzig.de 